\title{
Caracterización de pacientes con enfermedad del hígado graso no alcohólica en un hospital de alta complejidad, Colombia 2013
}

\section{Characterization of Patients with Non-Alcoholic fatty Liver Disease in a Highly Complex Colombian Hospital in 2013}

\author{
Claudia Marcela Vanegas R., MD, ${ }^{1}$ Camilo Restrepo R., MD, ${ }^{1}$ Natalia Vargas G., MD, ${ }^{1}$ Andrés Eduardo Marín C., MD, \\ Lina María Martínez S., MD, ${ }^{1}$ Carlos Enrique Yepes D., MD, ${ }^{2,3}$ Juan Carlos Restrepo G, MD. ${ }^{1,2,3}$
}

\author{
Universidad Pontificia Bolivariana, Escuela de \\ Ciencias de la Salud, Facultad de Medicina. Medellín, \\ Colombia \\ 2 Hospital Pablo Tobón Uribe, Grupo de Trasplante \\ de hígado. Universidad de Antioquia, Grupo de \\ Gastrohepatología. Medellín, Colombia \\ 3 Universidad Pontificia Bolivariana, Escuela de \\ Ciencias de la Salud, Facultad de Medicina. \\ Hospital Pablo Tobón Uribe, Grupo de trasplante \\ de hígado. Universidad de Antioquia, Grupo de \\ Gastrohepatología. Medellín, Colombia \\ e-mail: jcrestrepo@une.net.co; \\ jcrestrepo@hptu.org.co \\ Fecha recibido: $25-03-14$ \\ Fecha aceptado: $05-11-14$
}

\section{Resumen}

La enfermedad del hígado graso no alcohólica compromete de 2 a 8\% de la población general; esta se relaciona con obesidad, diabetes tipo 2 , síndrome metabólico y resistencia a la insulina.

Objetivo: caracterizar a los pacientes con enfermedad del hígado graso no alcohólica que consultaron al servicio de hepatología del hospital de 4to nivel, entre los años 2006 y 2011.

Metodología: estudio observacional descriptivo retrospectivo; la población estuvo constituida por la totalidad de los pacientes atendidos en el servicio de hepatología del hospital de 4to nivel, durante el periodo de estudio con diagnóstico de enfermedad del hígado graso no alcohólica, se excluyeron pacientes con hepatopatía de otro origen. La recolección de información se realizó por medio de las historias clínicas en un formulario de recolección de datos diseñado por los investigadores, luego fueron tabulados y analizados en el SPSS ${ }^{\circledR}$ versión 17.0. Se calcularon medidas de tendencia central, posición y dispersión para variables cuantitativas, frecuencias absolutas y relativas para las cualitativas. Se aplicaron las pruebas de U de MannWhitney y chi-cuadrado según correspondiera.

Resultados: el 58,5\% fueron pacientes de sexo femenino. Se encontró que 52,3\% del total de la muestra tenían sobrepeso según el índice de masa corporal y 72,1\% mostraron hígado graso por ecografía.

Conclusión: se encontró que la mayoría de los pacientes con enfermedad del hígado graso no alcohólica tienen elevado el índice de masa corporal, datos similares a los reportados en otras series.

\section{Palabras clave}

Hígado graso, resistencia a la insulina, obesidad.

\section{Abstract}

Non-alcoholic fatty liver disease (NAFLD) affects $2 \%$ to $8 \%$ of the general population. It is associated with obesity, type 2 diabetes, metabolic syndrome and insulin resistance.

Objective: The objective of this study was to characterize patients with NAFLD who were treated in the Hepatology Department of a 4th level hospital between 2006 and 2011.

Methods: This was a descriptive, observational and retrospective study of a population consisting of all patients treated in the hepatology department of a 4th level hospital who had been diagnosed with NAFLD. Patients with other liver diseases were excluded. Data was collected from medical records on a form designed by the researchers. Data were then tabulated and analyzed in SPSS ${ }^{\circledR}$ version 17.0. Measures of central tendency, position and dispersion were calculated for quantitative variables, and absolute and relative frequencies were calculated for qualitative variables. The Mann-Whitney $U$ test and the and chi-square test were used as appropriate.

Results: $58.5 \%$ of the sample were female patients. We found that $52.3 \%$ of the sample were overweight according to $\mathrm{BMI}$, and $72.1 \%$ were diagnosed with fatty liver by ultrasound.

Conclusion: We found that most patients with NASH have elevated body mass indices similar to reports published elsewhere.

\section{Keywords}

Fatty liver, insulin resistance, obesity. 


\section{INTRODUCCIÓN}

La enfermedad hepática no alcohólica consiste en la acumulación de grasa en los hepatocitos superior a 5\% del peso total del hígado (1). Es una entidad que tiene un amplio espectro clínico-patológico que incluye la esteatosis simple y la esteatohepatitis no alcohólica (EHNA); esta es cada vez más reconocida como una causa importante de morbilidad y mortalidad (2).

La prevalencia mundial presenta una gran variabilidad con cifras que van desde $2,8 \%$ a $88 \%$, dependiendo de la población estudiada.

Estos datos han ido aumentando proporcionalmente con las tasas de obesidad, sedentarismo y dietas altas en calorías (1). Se ha encontrado mayor predisposición en pacientes entre la cuarta y sexta década de la vida (3).

Además de la obesidad, la enfermedad hepática grasa no alcohólica también está asociada con el síndrome metabólico cuyos factores de riesgo incluyen resistencia a la insulina, diabetes, aumento del perímetro abdominal y dislipidemia (4).

Los hallazgos clínicos y de laboratorio iniciales suelen ser inespecíficos, pero tradicionalmente el diagnóstico se da por imágenes de manera incidental, principalmente por ecografía (5).

El mejor método para la identificación y clasificación del hígado graso es el histológico, el cual diferencia entre formas tempranas y tardías; sin embargo, para un adecuado diagnóstico se requiere la exclusión de otros trastornos, en particular de hepatitis viral, la ingesta significativa de alcohol, y la exposición a medicamentos potencialmente hepatotóxicos $(1,6)$.

Los cambios iniciales que se generan en la esteatohepatitis no alcohólica pueden predecir un futuro desarrollo de cirrosis, entre ellos la elevación de las aminotransferasas, por lo tanto la identificación temprana de estos puede permitir su prevención (7). Los seguimientos hasta por diez años han demostrado que los pacientes con EHNA pueden progresar a cirrosis en $25 \%$ de casos, con una mortalidad hepática de $12 \%$. Muchos de los pacientes diagnosticados con cirrosis criptogénica (hasta $80 \%$ ) eventualmente tenían EHNA, y cuando son trasplantados frecuentemente recidivan en el hígado trasplantado con cambios idénticos a EHNA (8).

Es por esta razón que cambios en el estilo de vida que generen disminución en el peso corporal pueden modificar los niveles de aminotransferasas, y tener algún efecto final sobre la histología hepática y el curso natural de la enferme$\operatorname{dad}(2,9,10)$.

Idrovo y Guevara (11) publicaron que la prevalencia estimada de enfermedad del hígado graso no alcohólico (HGNA) es de $20 \%$ en la población general, constituyéndose en el principal motivo de consulta en hepatología, mientras la prevalencia de EHNA es de 2\% a 3\%. Pacientes con elevación crónica de aminotransferasas pueden tener una prevalencia HGNA hasta de $21 \%$ a $63 \%$, y si son obesos hasta de $95 \%$. La prevalencia de EHNA en obesos es de $25 \%$ y en diabéticos tipo II es de 63\%. Lo anterior da cuenta de la necesidad de realizar este trabajo que tuvo como objetivo caracterizar a los pacientes con enfermedad hepática grasa no alcohólica que consultaron al servicio de hepatología de un hospital de alta complejidad entre los años 2006 y 2011.

\section{METODOLOGÍA}

Se llevó a cabo un estudio de tipo observacional descriptivo retrospectivo; la población estuvo constituida por pacientes atendidos en el servicio de hepatología de un hospital de alta complejidad de la ciudad de Medellín, durante el periodo de estudio y que tenían una enfermedad del hígado graso no alcohólica. Los criterios de exclusión fueron: paciente alcohólico o con antecedente de consumo constante de alcohol $>40 \mathrm{~g} /$ día, paciente con diagnóstico de hepatitis viral o autoinmune, trastornos metabólicos, factores hereditarios, y/o drogas o toxinas. El plan de recolección de información consistió en tomar las historias clínicas de los pacientes y se tomaron los datos clínicos, paraclínicos y de tratamiento relacionados con la evolución clínica de la enfermedad en estudio, por medio de un formulario de recolección de datos, luego estos fueron tabulados y analizados en el paquete estadístico SPSS versión 17.0. A las variables cuantitativas se les calculó medidas de tendencia central, posición y dispersión, y a las cualitativas frecuencias absolutas y se estimó la prevalencia de la enfermedad en cuestión, se realizó análisis de asociación entre el hígado graso y el IMC, usando las pruebas estadísticas requeridas según fue la distribución de los datos. La investigación contó con la aprobación del Comité de Ética de la institución y se guardó la confidencialidad de los datos que contenían los archivos clínicos.

\section{RESULTADOS}

En el estudio se incluyeron 294 pacientes, de los cuales $58,5 \%$ pertenecían al género femenino, la edad promedio fue de 48 años (DS 13,7), hasta 50\% de los pacientes tenían una edad $\leq 50$ años. En cuanto a las variables antropométricas, el IMC promedio (DS) fue de 28,7 (5,0); 52,3\% de los pacientes fueron clasificados con sobrepeso según el IMC, 18,1\% con obesidad grado I, $8,3 \%$ con obesidad grado II y $3,2 \%$ con obesidad mórbida; solo $18,1 \%$ se encontraba con IMC normal; es necesario aclarar que estos datos hacen referencia a $73,5 \%$ de los pacientes con la información disponible.

Para las pruebas de laboratorio, se observó que hasta 50\% de los pacientes tenía valores de aspartato amino transfe- 
rasa (AST) y alanino amino transferasa (ALT) menores o iguales a 39 y $54 \mathrm{UI} / \mathrm{L}$ respectivamente y para la fosfatasa alcalina (FA) de 94 UI/L. Para los valores de colesterol total y triglicéridos, se observó que hasta 50\% de los pacientes tenía valores menores o iguales a $205 \mathrm{mg} / \mathrm{dl}$ y $164 \mathrm{mg} / \mathrm{dl}$ respectivamente. La mediana para la ferritina fue de 192 $\mathrm{ng} / \mathrm{ml}$ con un promedio de 311,3 (DS 330,6) (tabla 1).

Tabla 1. Pruebas de laboratorio de los pacientes atendidos en el servicio de hepatología en el hospital de alta complejidad.

\begin{tabular}{lcccccc}
\hline \multicolumn{1}{c}{ Examen } & N & Media & Mediana & DS & Min & Max \\
\hline AST (UI/L) & 263 & 55,08 & 39,0 & 70,162 & 14 & 718 \\
ALT (UI/L) & 257 & 76,25 & 54,0 & 84,694 & 10 & 1057 \\
GGT (UI/L) & 161 & 96,03 & 63,0 & 116,979 & 15 & 1025 \\
$\begin{array}{l}\text { Bilirrubina total } \\
\text { (mg/dl) }\end{array}$ & 173 &, 774 &, 600 &, 8347 &, 2 & 10,0 \\
$\begin{array}{l}\text { Bilirrubina directa } \\
\text { (mg/dl) }\end{array}$ & 107 &, 287 &, 200 &, 3539 &, 0 & 2,1 \\
$\begin{array}{l}\text { Fosfatasa alcalina } \\
\text { (Ul/l) }\end{array}$ & 189 & 122,92 & 94,0 & 135,954 & 3 & 1298 \\
$\begin{array}{l}\text { Colesterol total } \\
\text { (mg/dl) }\end{array}$ & 196 & 210,81 & 205,0 & 46,452 & 115 & 384 \\
HDL (mg/dl) & 91 & 43,79 & 42,0 & 11,762 & 18 & 82 \\
LDL (mg/dl) & 59 & 144,01 & 138,0 & 59,666 & 61 & 505 \\
Triglicéridos (mg/dl) & 189 & 190,28 & 164,0 & 105,548 & 60 & 790 \\
Ferritina (ng/ml) & 123 & 311,3 & 192,0 & 330,6 & 1,60 & 1976,0 \\
\hline
\end{tabular}

Las comorbilidades más frecuentes fueron dislipidemia con $32,1 \%$, seguida de hipertensión arterial (HTA) con 29,3\% y diabetes mellitus tipo 2 (DM2) con 15,6\%, otras comorbilidades se presentaron en menor frecuencia (tabla 2).

Tabla 2. Comorbilidades de los pacientes atendidos en el servicio de hepatología en el hospital de alta complejidad.

\begin{tabular}{lc}
\hline \multicolumn{1}{c}{ Comorbilidades } & $\%$ \\
\hline Dislipidemia & $32,1 \%$ \\
Hipertensión arterial & $29,3 \%$ \\
Diabetes mellitus tipo 2 & $15,6 \%$ \\
Hipotiroidismo & $11,9 \%$ \\
Neoplasia & $3,1 \%$ \\
Hepatitis viral VHB & $1,4 \%$ \\
Lupus eritematoso sistémico & $1,4 \%$ \\
Hepatitis viral VHC & $0,7 \%$ \\
Infarto agudo de miocardio & $0,7 \%$ \\
Accidente cerebrovascular & $0,3 \%$ \\
Otra & $42,2 \%$ \\
\hline
\end{tabular}

Respecto al consumo de alcohol, 33,3\% de los pacientes no consumía alcohol y $26,9 \%$ lo hacía de forma ocasional pero menos de $40 \mathrm{~g} /$ día, es necesario aclarar que no se tuvo información de $39,8 \%$ de los registros médicos.

Según el reporte de la ecografía, $72,1 \%$ tenía hígado graso, $0,7 \%$ hepatomegalia y $5,4 \%$ sin hígado graso; se aclara que $21,8 \%$ de los reportes no se encontraron en las historias clínicas. Con respecto al reporte de la biopsia hepática se encontró que solo $19 \%$ contaba con el resultado, de estos, $17,1 \%$ tuvo confirmación de hígado graso. El porcentaje restante $(81 \%)$ no contaba con este resultado.

En la figura 1 se observa cómo hasta $50 \%$ los pacientes con hígado graso tenía un IMC $\leq 27 \mathrm{~kg} / \mathrm{m}^{2}$, con un rango intercuartílico de mayor precisión. En la tabla 3 se observa que el diagnóstico de hígado graso fue mayor en todos los casos de los pacientes clasificados con IMC alterado en sus diferentes clasificaciones, siendo $100 \%$ para los pacientes con obesidad mórbida (tabla 3 ).

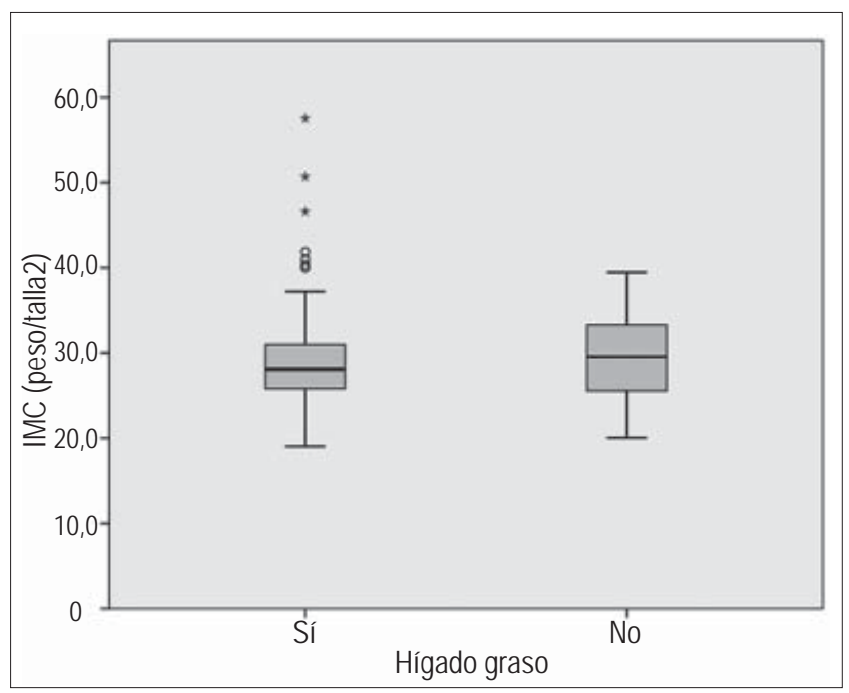

Figura 1. IMC vs. hígado graso de los pacientes atendidos en el servicio de hepatología en el hospital de alta complejidad.

\section{DISCUSIÓN}

Las HGNA/EHNA son reconocidas en la actualidad como importantes problemas en salud pública por su relevante asociación con obesidad y DM. Estas son consideradas como causa principal de enfermedad hepática crónica, las cuales pueden culminar con la aparición de cirrosis, cirrosis descompensada y hepatocarcinoma, generando gran impacto sobre la morbimortalidad de las personas y grandes costos para el sistema de salud (12). Las mujeres no están libres de esta entidad, de allí la importancia de aprender a reconocer y tratarla de una forma oportuna y apropiada. En el estudio realizado por Leite y col se encontraron datos similares a los del presente estudio como la frecuencia del género femenino con $68,1 \%$ (7). La HGNA/EHNA es una 
entidad con una alta prevalencia, con una relación primaria con el síndrome de resistencia a la insulina y de la cual es la manifestación hepática. Se debe reconocer la enfermedad en pacientes con factores de riesgo y se debe estimular tempranamente unos cambios saludables del estilo de vida que se mantengan en el tiempo y que permitan la resolución sin progresión a cirrosis.

Tabla 3. IMC vs. hígado graso de los pacientes atendidos en el servicio de hepatología en el hospital de alta complejidad.

\begin{tabular}{llcccc}
\hline & & \multicolumn{3}{c}{ Hígado graso } & \multirow{2}{*}{ Total } \\
\cline { 3 - 4 } & & & Si & No & \\
\hline Grupo de clasificación & Normal & $\mathrm{n}$ & 33 & 2 & 35 \\
IMC Kg/m² & & $\%$ & $94,3 \%$ & $5,7 \%$ & $100,0 \%$ \\
& Sobrepeso & $\mathrm{n}$ & 90 & 6 & 96 \\
& & $\%$ & $93,8 \%$ & $6,3 \%$ & $100,0 \%$ \\
& Obesidad I & $\mathrm{n}$ & 32 & 3 & 35 \\
& & $\%$ & $91,4 \%$ & $8,6 \%$ & $100,0 \%$ \\
& Obesidad II & $\mathrm{n}$ & 13 & 3 & 16 \\
& & $\%$ & $81,3 \%$ & $18,8 \%$ & $100,0 \%$ \\
& Obesidad & $\mathrm{n}$ & 7 & 0 & 7 \\
& mórbida & $\%$ & $100,0 \%$ & $0,0 \%$ & $100,0 \%$ \\
\hline Total & & $\mathrm{n}$ & 175 & 14 & 189 \\
& & $\%$ & $92,6 \%$ & $7,4 \%$ & $100,0 \%$ \\
\hline
\end{tabular}

En los últimos años se ha avanzado en el conocimiento de la historia natural de la HGNA/EHNA, aunque existen ciertas limitaciones para los estudios, básicamente el curso lento progresivo de la enfermedad hepática, con una buena parte de los estudios de corto a mediano seguimiento y la posibilidad de sesgo de referencia y de selección. La esteatosis hepática está fuertemente relacionada a todos los fenómenos clínicos de resistencia a la insulina (IR), es decir, obesidad, síndrome metabólico y DM (13). Cuando se realizan estudios en población con obesidad (IMC $>30 \mathrm{~kg} / \mathrm{m}^{2}$ ), 50 a $90 \%$ puede cursar con HGNA/EHNA. En un estudio se encontró que hasta 50\% los pacientes con hígado graso tenía un índice de masa corporal menor o igual a 27, que fueron clasificados en sobrepeso y en cuanto a la obesidad con $27,5 \%$ (7). Se considera que hasta $50 \%$ de los diabéticos se encuentra con esteatosis grasa, una relación muy importante que también se ha asociado como un factor pronóstico relevante. A pesar de no estar incluida dentro de los criterios diagnósticos la esteatosis hepática está considerada como la manifestación en el hígado del síndrome metabólico, y se ha demostrado que a mayor número de criterios presentes en una persona hay mayor riesgo de HGNA.

En una gran proporción los pacientes son hallados con niveles elevados de transaminasas en exámenes de control en programas de riesgo cardiovascular por sus patologías de base (14). El aumento de enzimas generalmente es leve, menos de 3 veces los valores de referencia, con una relación AST/ALT < 1 (en contraste a la hepatopatía alcohólica) (15). En nuestro estudio se observan valores superiores respecto a los encontrados en el estudio de Ortiz-López y col con resultados de la media de $41 \pm 2 \mathrm{UI} / \mathrm{L}$ y $59 \pm 3 \mathrm{UI} / \mathrm{L}$ para el AST y ALT respectivamente (16). Los pacientes con esteatosis y con enzimas hepáticas normales o con un cuadro establecido de cirrosis, no requieren biopsia hepática por el poco valor que puede ofrecer y los riesgos que de todas maneras genera un estudio invasivo (17). Aunque solo a $19 \%$ de los pacientes participantes se les realizó biopsia hepática, $91 \%$ de estos tuvo hallazgos confirmatorios de hígado graso, en comparación con el estudio realizado por Bhat y col en el que a 53\% de la población se le realizó biopsia con cambios histopatológicos en $100 \%$ de estos (2). Una de las limitaciones del estudio es la dificultad para conseguir la totalidad de los datos de los pacientes del estudio, por el diligenciamiento incompleto de algunas de las historias clínicas, o la falta de estandarización para el diagnóstico de la enfermedad. En el futuro sería necesaria la elaboración de nuevos estudios en relación a la patogenia de la HGNA para complementar los hallazgos de este estudio. En conclusión, se encontró que la mayoría de los pacientes con enfermedad del hígado graso no alcohólica tiene elevado el índice de masa corporal, datos similares a los reportados en otras series.

\section{REFERENCIAS}

1. Souza MR, Diniz M de F, Medeiros-Filho JE, Araújo MS. Metabolic syndrome and risk factors for non-alcoholic fatty liver disease. Arq Gastroenterol 2012; 49(1): 89-96.

2. Bhat G, Baba CS, Pandey A, Kumari N, Choudhuri G. Life style modification improves insulin resistance and liver histology in patients with non-alcoholic fatty liver disease. World J Hepatol 2012; 4(7): 209-17.

3. Bellentani S, Marino M. Epidemiology and natural history of non-alcoholic fatty liver disease (NAFLD). Ann Hepatol 2009; 8(Suppl. 1): S4-8.

4. Santos LF, Hernández G, Varón A, Beltrán O, Botero RC, Mejía G. Enfermedad hepática por infiltración grasa no alcohólica: La nueva pandemia del milenio. Rev Col Gastroenterol 2010; 25(4): 380-398.

5. Roldan-Valadez E, Favila R, Martínez-López M, Uribe M, Méndez-Sánchez N. Imaging techniques for assessing hepatic fat content in nonalcoholic fatty liver disease. Ann Hepatol 2008; 7(3): 212-20.

6. Farrell GC, van Rooyen D, Gan L, Chitturi S. NASH is an Inflammatory Disorder: Pathogenic, Prognostic and Therapeutic Implications. Gut Liver 2012; 149-71.

7. Leite AB, Mattos AA, Mattos AZ, Coral GP, Evaldt S. Risk factors for nonalcoholicsteatohepatitis in cryptogenic cirrhosis. Arq Gastroenterol 2012; 49(4): 245-9. 
8. Cubides VI, Guevara LG. Enfermedad hepática grasa no alcohólica: NAFLD. Rev Col de Gastroenterol 2004; 19(1): 44-49.

9. Jun DW. [The role of diet in non-alcoholic fatty liver disease]. Korean J Gastroenterol 2013; 61(5): 243-51.

10. Centis E, Moscatiello S, Bugianesi E, Bellentani S, Fracanzani AL, Calugi S. Stage of change and motivation to healthier lifestyle in non-alcoholic fatty liver disease. J Hepatol 2013; 58(4): 771-7.

11. Idrovo V, Guevara G. Enfermedad hepática grasa no alcohólica: NAFLD. Rev Colomb Gastroenterol 2004; 19 (1): 44-9.

12. Lam B, Younossi ZM. Treatment options for nonalcoholic fatty liver disease. Therap Adv Gastroenterol 2010; 3(2): 121-137.

13. Farell GC, George J, Hall P, McCullough AJ. Fatty Liver Disease: NASH and Related Disorders. NEJM 2005; 353(20): 2200-2201.
14. Suzuki A, Angulo P, Lymp J, St Sauver J, Muto A, Okada T, Lindor K. Chronological development of elevated aminotransferases in a nonalcoholic population. Hepatology 2005; 41(1): 64-71.

15. Angulo P. Nonalcoholic Fatty Liver Disease. N Engl J Med 2002; 346(16): 1221-31.

16. Ortiz-Lopez C, Lomonaco R, Orsak B, Finch J, Chang Z, Kochunov VG, Hardies J, Cusi K. Prevalence of prediabetes and diabetes and metabolic profile of patients with nonalcoholic fatty liver disease (NAFLD). Diabetes Care 2012; 35(4): 873-8.

17. Hashimoto E, Taniai M, Tokushige K. Characteristics and diagnosis of NAFLD/NASH. J Gastroenterol Hepatol 2013; 28 (Suppl. 4): 64-70. 